\title{
Role of miR-139-5p in ectopic endometrial stromal cells and the underlying molecular mechanism
}

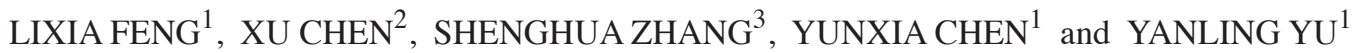 \\ ${ }^{1}$ Department of Gynecology, The Second Affiliated Hospital of Shenzhen University, Shenzhen, Guangdong 518100; \\ ${ }^{2}$ Department of Obstetrics and Gynecology, Huashan Hospital North Affiliated to Fudan University, \\ Shanghai 210907; ${ }^{3}$ Department of Obstetrics and Gynecology, The Second Hospital of \\ Anhui Medical University, Hefei, Anhui 230601, P.R. China
}

Received December 19, 2019; Accepted June 8, 2021

DOI: $10.3892 /$ etm.2021.10686

\begin{abstract}
Endometriosis is an estrogen-dependent disease. Studies have shown that miR-139-5p is significantly upregulated in endometriosis lesions, but its specific role and molecule mechanism in endometriosis has not yet been reported. The malignant biological behavior of ectopic endometrial stromal cells (ESCs) is similar to that of malignant cancer cells. BBC3 (BCL2 binding component 3) is a known apoptosis inducer and it serves key roles in the regulation of cell behavior. However, the role of BBC3 in ectopic ESCs remains unknown. The present study aimed to investigate the role of miR-139-5p in the progression of endometriosis and to determine its underlying molecular mechanism of action. Ectopic, non-ectopic and normal endometrial stromal cells (ESCs) were extracted from endometrial samples, and reverse transcription-quantitative PCR was performed to determine microRNA (miR)-139-5p and Bcl-2 binding component 3 (BBC3) mRNA expression levels in endometrial tissue samples and ESCs. The target gene of miR-139-5p was predicted using TargetScan software and verified using a dual luciferase reporter assay. Western blotting was performed to determine BBC3 protein expression levels. Flow cytometry analysis, and MTT and Transwell assays were performed to assess cell apoptosis, viability, and migration and invasion of the cells transfected with inhibitor control, miR-139-5p inhibitor, miR-139-5p inhibitor + control-small interfering (si)RNA or miR-139-5p inhibitor + BBC3-siRNA, respectively. The results demonstrated that miR-139-5p expression levels were upregulated in ectopic endometrial samples and ESCs compared with the respective control groups. Furthermore, it was verified that BBC3 was a direct target of miR-139-5p, and both BBC3 mRNA and protein expression
\end{abstract}

Correspondence to: Mrs. Yanling Yu, Department of Gynecology, The Second Affiliated Hospital of Shenzhen University, 118 Longjing Second Road, Shenzhen, Guangdong 518100, P.R. China E-mail: yuyanling183719@163.com

Key words: microRNA-139-5p, endometriosis, ectopic endometrial stromal cells, Bcl-2 binding component 3 levels were downregulated in ectopic endometrial samples and ESCs. Both transfection with the miR-139-5p inhibitor and BBC3-small interfering (si)RNA markedly downregulated miR-139-5p and BBC3 expression levels in ectopic ESCs, respectively. miR-139-5p inhibitor-induced upregulated $\mathrm{BBC} 3$ expression was reversed following transfection with BBC3-siRNA. Furthermore, the miR-139-5p inhibitor significantly decreased viability, migration and invasion, while inducing apoptosis in ectopic ESCs compared with the inhibitor control group. Notably, the aforementioned effects were reversed by knocking down BBC3 expression. In conclusion, the results of the present study suggested that miR-139-5p may play a key role in the progression of endometriosis by regulating the viability of ESCs and directly targeting BBC3.

\section{Introduction}

Endometriosis is an estrogen-dependent condition that is characterized by the ectopic growth of endometrial tissue, which can result in symptoms such as pelvic pain and dysmenorrhea and problems such as infertility $(1,2)$. Although endometriosis is known to be a benign disease, it exhibits characteristics common of malignant tumors, such as infinite growth, angiogenesis, infiltration and destruction of surrounding tissues, and local or distant metastasis $(3,4)$. Furthermore, the migratory and invasive properties of ectopic endometrial stromal cells (ESCs) were found to be similar to those of malignant cancer cells (5). However, to the best of our knowledge, the molecular mechanisms underlying the proliferation of ectopic ESCs remain unclear, thus further studies on endometriosis are required to accelerate the development of effective treatment strategies.

MicroRNAs (miRNAs/miRs) are small, non-coding RNA molecules of 20-22 nucleotides in length, which are involved in cell proliferation and regulating the expression of target genes $(6,7)$. Previous studies have suggested that miRNAs were associated with several types of human disease, including cancer, and cardiovascular, gynecological and inflammatory diseases (8-11). Prislei et al (12) demonstrated that miR-200c affected the proliferation of ovarian cancer cell lines by regulating tubulin $\beta 3$ class III expression. Furthermore, He et al (13) confirmed the role of miR-155 in pathological 
cardiac remodeling, as it was discovered that miR-155-induced targeting of tumor protein p53-inducible nuclear protein 1 improved pathological cardiac remodeling (13). Previous studies have indicated that several types of miRNA participate in the progression of endometriosis, as the expression profiles of miRNAs were demonstrated to be different (abnormal decrease and increase in contrast) between non-ectopic and ectopic endometrial tissues $(14,15)$. Of these, miR-139-5p has been demonstrated to play key roles in several types of disease. For example, Song et al (16) reported that miR-139-5p was a potential tumor suppressor and prognostic factor for colorectal cancer. Furthermore, miR-139-5p was shown to play a regulatory role in numerous types of disease, such as neurological diseases, atherosclerosis and endometriosis (17-19). Therefore, it is suggested that miR-139-5p plays important roles in the regulation of cellular functions. In addition, a previous study reported that miR-139-5p expression was significantly upregulated in ectopic stroma and ectopic stroma cells (19); however, to the best of our knowledge, its underlying molecular mechanism remains unclear. As ESCs have been found to play crucial roles in endometriosis, we hypothesized that miR-139-5p may be involved in endometriosis by regulating ESC function.

The present study aimed to investigate whether miR-139-5p served a role in endometriosis by regulating the malignant behaviors (apoptosis, viability, migration and invasion) of ectopic ESCs. Furthermore, the study sought to determine its molecular mechanism of action to provide novel therapeutic strategies for endometriosis.

\section{Materials and methods}

Patient samples. The present study was approved by the Ethics Committee of The Second Affiliated Hospital of Shenzhen University (Shenzhen, China) and written informed consent was provided by all participants prior to the study start. A total of 15 paired ectopic and non-ectopic endometrial tissue samples were collected from premenopausal women (mean age, 30 years; age range: 26-36 years) who underwent gynecological procedures for benign indications at The Second Affiliated Hospital of Shenzhen University from April 2018 to April 2019. Normal endometrial tissue samples were collected from 15 premenopausal women (age range, 26-36 years) with hysteromyoma at The Second Affiliated Hospital of Shenzhen University (Shenzhen Baoan District People's Hospital; Shenzhen, China) from April 2018 to April 2019 and used as the control samples. The inclusion criteria for patients with endometriosis were as follows: i) Patients in the mid-to-late proliferative phase of the disease; and ii) patients who had not received hormonal treatment 3 months prior to surgery. Patients who had malignancy or other diseases, such as submucous myoma, endometrial hyperplasia, adenomyosis or chronic pelvic inflammation were excluded from the present study.

Extraction and culture of normal, non-ectopic and ectopic ESCs. Non-ectopic ESCs were isolated from non-ectopic endometrial tissue, ectopic ESCs were isolated from ectopic endometrial tissue and the normal ESCs were isolated from normal tissue samples. Tissues samples were washed three times with PBS and dissected, prior to digestion with $0.1 \%$ type IV collagenase solution and $0.25 \%$ trypsin at $37^{\circ} \mathrm{C}$ for
2 h. Subsequently, normal, non-ectopic and ectopic ESCs were extracted from tissue samples as previously described (20), and cultured in Ham's F12/DMEM (Gibco; Thermo Fisher Scientific, Inc.) supplemented with 10\% FBS (Gibco; Thermo Fisher Scientific, Inc.) and $1 \%$ penicillin/streptomycin (Gibco; Thermo Fisher Scientific, Inc.) at $37^{\circ} \mathrm{C}$ for $24 \mathrm{~h}$ in $5 \% \mathrm{CO}_{2}$. The purity of the ESCs was determined following the third passage, and ESCs with $>99 \%$ purity were selected for subsequent experimentation.

Reverse transcription-quantitative $(R T-q) P C R$. Total RNA was extracted from normal, non-ectopic and ectopic ESCs and endometrial tissues using RNAqueous ${ }^{\mathrm{TM}}-96$ Total RNA Isolation kit (Invitrogen; Thermo Fisher Scientific, Inc.). Total RNA was reverse transcribed into first strand cDNA using a RevertAid First Strand cDNA Synthesis kit (Invitrogen; Thermo Fisher Scientific, Inc.), according to the manufacturer's protocol. qPCR was subsequently performed on a Prism 7000 Real-Time PCR Detection system (Applied Biosystems; Thermo Fisher Scientific, Inc.) using a SYBR qPCR Master Mix (Vazyme Biotech Co., Ltd.), according to the manufacturer's protocol. The primer sequences used for the qPCR were designed by Sangon Biotech Co., Ltd. and were as follows: GAPDH forward, 5'-CTTTGGTATCGTGGAAGGACTC-3' and reverse, 5'-GTAGAGGCAGGGATGATGTTCT-3'; U6 forward, 5'-GCTTCGGCAGCACATATACTAAAAT-3' and reverse, 5'-CGCTTCACGAATTTGCGTGTCAT-3'; miR-139-5p forward, 5'-AGCGGCATGATCAACATTTGC TAAAG-3' and reverse, 5'-TCTGTATGGTTTTCCTTCCGG CTT-3'; BBC3 forward, 5'-ACGACCTCAACGCGCAGTA-3' and reverse, 5'-CTAGTTGGGCTCCATTTCTGG-3'. The following thermocycling conditions were used for the qPCR: Initial denaturation at $95^{\circ} \mathrm{C}$ for $5 \mathrm{~min}$; followed by 40 cycles of $95^{\circ} \mathrm{C}$ for $10 \mathrm{sec}, 60^{\circ} \mathrm{C}$ for $30 \mathrm{sec}$ and $72^{\circ} \mathrm{C}$ for $30 \mathrm{sec}$. Relative mRNA expression levels of miR-139-5p and Bcl-2 binding component 3 (BBC3) were quantified using the $2^{-\Delta \Delta \mathrm{Cq}}$ method (21) and normalized to the internal reference genes, U6 or GAPDH, respectively. All experiments were performed in triplicate.

Cell transfection. Ectopic ESCs were seeded into 6-well plates at a density of $5 \times 10^{4}$ cells $/ \mathrm{ml}$ and cultured at $37^{\circ} \mathrm{C}$ overnight. A total of $50 \mathrm{nM}$ inhibitor control (5'-GGACCA AATCTCGAGATTTGG-3'; Shanghai GenePharma, Co., Ltd.), 50 nM miR-139-5p inhibitor (5'-ACTGGAGACACG TGCACTGTAGA-3'; Shanghai GenePharma, Co., Ltd.), $1 \mu \mathrm{M}$ control-small interfering (si)RNA (cat. no. sc-36869; Santa Cruz Biotechnology, Inc.), $1 \mu \mathrm{M}$ BBC3-siRNA (cat. no. sc-37153; Santa Cruz Biotechnology, Inc.), $50 \mathrm{nM}$ miR-139-5p inhibitor $+1 \mu \mathrm{M}$ control-siRNA or $50 \mathrm{nM}$ miR-139-5p inhibitor $+1 \mu \mathrm{M}$ BBC3-siRNA were transfected into ectopic ESCs using Lipofectamine ${ }^{\circledR} 2000$ reagent (Invitrogen; Thermo Fisher Scientific, Inc.), according to the manufacturer's protocol. Following $48 \mathrm{~h}$ of transfection at $37^{\circ} \mathrm{C}$, cells were collected (centrifugation at $4^{\circ} \mathrm{C}$ at $12,000 \mathrm{x} \mathrm{g}$ for $15 \mathrm{~min}$ ) and the transfection efficiency was determined using RT-qPCR. Subsequent experiments were performed $48 \mathrm{~h}$ after transfection.

miRNA target analysis and dual luciferase reporter assay. The binding relationship between miR-139-5p and BBC3 was 
determined using TargetScan software (version 7.1; www. targetscan.org/vert_71). The 3'-untranslated region (UTR) of BBC3 that contained the target sequence of miR-139-5p was attained via RT-qPCR and integrated into the pmirGLO vector (Promega Corporation) to construct the wild-type (WT)-BBC3 reporter vector. Similarly, a mutant (MUT)-BBC3 reporter vector was also constructed using a QuikChange Site-Directed Mutagenesis kit (Stratagene; Agilent Technologies, Inc.) according to the manufacturer's instructions. Following incubation at $37^{\circ} \mathrm{C}$ for $24 \mathrm{~h}, 293 \mathrm{~T}$ cells (ATCC) were co-transfected with WT-BBC3 or MUT-BBC3 luciferase reporter vectors and miR-139-5p mimic (5'-UCUACAGUG CACGUGUCUCCAG-3'; Shanghai GenePharma Co., Ltd.) or mimic control (5'-UCUCCGAACGUGUCACGU-3'; Shanghai GenePharma Co., Ltd.) using Lipofectamine 2000 at $37^{\circ} \mathrm{C}$ for $48 \mathrm{~h}$. Following the transfection, firefly and Renilla luciferase activities were detected using a Dual Luciferase Reporter assay system (Promega Corporation), according to the manufacturer's protocol. Firefly luciferase activity was normalized to Renilla luciferase activity. All experiments were performed in triplicate.

Western blotting. Total protein from endometrial tissues and ESCs was extracted using a Radio Immunoprecipitation Assay (RIPA) Lysis Solution (Beyotime Institute of Biotechnology; cat. no. P0013B) and quantified using a BCA assay kit (Pierce; Thermo Fisher Scientific, Inc.). Total protein (40 $\mu \mathrm{g} / \mathrm{lane})$ was separated via $10 \%$ SDS-PAGE and then transferred onto a polyvinylidene difluoride membrane. Membranes were blocked with $5 \%$ non-fat milk at room temperature for $1 \mathrm{~h}$ and subsequently incubated with primary antibodies against BBC3 (1:1,000; cat. no. ab9643; Abcam) and GAPDH (1:1,000; cat. no. ab9485; Abcam) overnight at $4^{\circ} \mathrm{C}$. Following the primary antibody incubation, the membranes were washed three times with PBS-0.1\% Tween-20 and incubated with an HRP-conjugated secondary antibody (1:2,000; cat. no. 7074; Cell Signaling Technology, Inc.) at room temperature for $1 \mathrm{~h}$. Protein bands were visualized using enhanced chemiluminescence (Pierce; Thermo Fisher Scientific, Inc.), according to the manufacturer's protocol. All experiments were performed in triplicate.

MTT assay. Following transfection for $48 \mathrm{~h}$ at $37^{\circ} \mathrm{C}$, ectopic ESCs were seeded into 96-well plates and incubated at $37^{\circ} \mathrm{C}$ overnight. Subsequently, $10 \mu 1$ MTT solution (Beyotime Institute of Biotechnology) was added to each well and incubated at $37^{\circ} \mathrm{C}$ for a further $4 \mathrm{~h}$. Following the MTT incubation, the purple formazan crystals were dissolved in $100 \mu \mathrm{l}$ DMSO and cell viability was subsequently analyzed at a wavelength of $490 \mathrm{~nm}$ using a microplate reader (Bio-Rad Laboratories, Inc.). Relative cell viability of each experimental group was normalized to the control group using optical density values. All experiments were performed in triplicate.

Flow cytometric analysis of apoptosis. Following transfection for $48 \mathrm{~h}$ at $37^{\circ} \mathrm{C}$, ectopic ESCs were seeded into 6-well plates at $37^{\circ} \mathrm{C}$ overnight, collected via trypsinization by centrifugation at $1,000 \times \mathrm{g}$ at $4^{\circ} \mathrm{C}$ for $5 \mathrm{~min}$, washed with PBS and then re-suspended in $1 \mathrm{X}$ binding buffer (Beyotime Institute of Biotechnology) from the Annexin-V/propidium iodide (PI)
Apoptosis Detection kit according to the manufacturer's protocol. A total of $100 \mu \mathrm{l}$ cell suspension was transferred into a $5 \mathrm{ml}$ tube, and cells were respectively stained with $5 \mu \mathrm{l}$ Annexin V-FITC and $5 \mu \mathrm{l}$ PI (Beyotime Institute of Biotechnology) at room temperature in the dark for $15 \mathrm{~min}$, according to the manufacturer's protocol. Apoptotic cells (the percentage of early + late apoptotic cells) were analyzed using a FACSCalibur flow cytometer (BD Biosciences) and the data were analyzed using FlowJo software (version 7.6.1; FlowJo LLC). All experiments were performed in triplicate.

Transwell assays. For the cell invasion and migration assays, following transfection for $48 \mathrm{~h}$ at $37^{\circ} \mathrm{C}$, ectopic ESCs $\left(2 \times 10^{4}\right.$ cells/well) were plated into the upper chambers of Transwell plates in serum-free DMEM (Gibco; Thermo Fisher Scientific, Inc.). Transwell membranes were precoated with Matrigel (BD Biosciences) at $37^{\circ} \mathrm{C}$ for $30 \mathrm{~min}$ for the invasion assay only. DMEM supplemented with $10 \%$ FBS was plated into the lower chambers. Following incubation at $37^{\circ} \mathrm{C}$ for $24 \mathrm{~h}$, the migratory or invasive cells in the lower chambers were fixed with $4 \%$ paraformaldehyde at room temperature for 30 min and subsequently stained with $0.1 \%$ crystal violet at room temperature for $15 \mathrm{~min}$. Stained cells were counted in five randomly selected fields of view using a light microscope (magnification, x100). All experiments were performed in triplicate.

Statistical analysis. Statistical analysis was performed using SPSS software (version 18.0; SPSS, Inc.). Data are presented as the mean \pm SD. Statistical differences between two groups were assessed using an unpaired Student's t-test (for luciferase activity analysis), while a one-way ANOVA followed by a Tukey's post hoc test was used for multiple comparisons. $\mathrm{P}<0.05$ was considered to indicate a statistically significant difference. All experiments were performed in triplicate.

\section{Results}

miR-139-5p expression levels are upregulated in and ectopic endometriosis tissues and ESCs. miR-139-5p expression levels in patients with or without endometriosis were detected via RT-qPCR. The results demonstrated that miR-139-5p expression was significantly upregulated in ectopic endometrial samples compared with the normal endometrium samples (Fig. 1A). Subsequently, miR-139-5p expression levels were assessed in ESCs, and the results revealed that the expression levels of miR-139-5p were significantly upregulated in ectopic ESCs compared with normal ESCs (Fig. 1B).

$B B C 3$ is a direct target of miR-139-5p. To determine the underlying molecular mechanisms of miR-139-5p in endometriosis, its candidate targets were predicted using TargetScan software, which identified BBC3 as a potential target of miR-139-5p (Fig. 2A). A dual luciferase reporter assay was subsequently performed in $293 \mathrm{~T}$ cells to identify the binding sites of miR-139-5p in the BBC3 3'-UTR. The results demonstrated that the co-transfection with the miR-139-5p mimic significantly decreased the relative luciferase activity of the WT-BBC3 3'-UTR compared with co-transfection with the mimic control; however, no significant differences were 

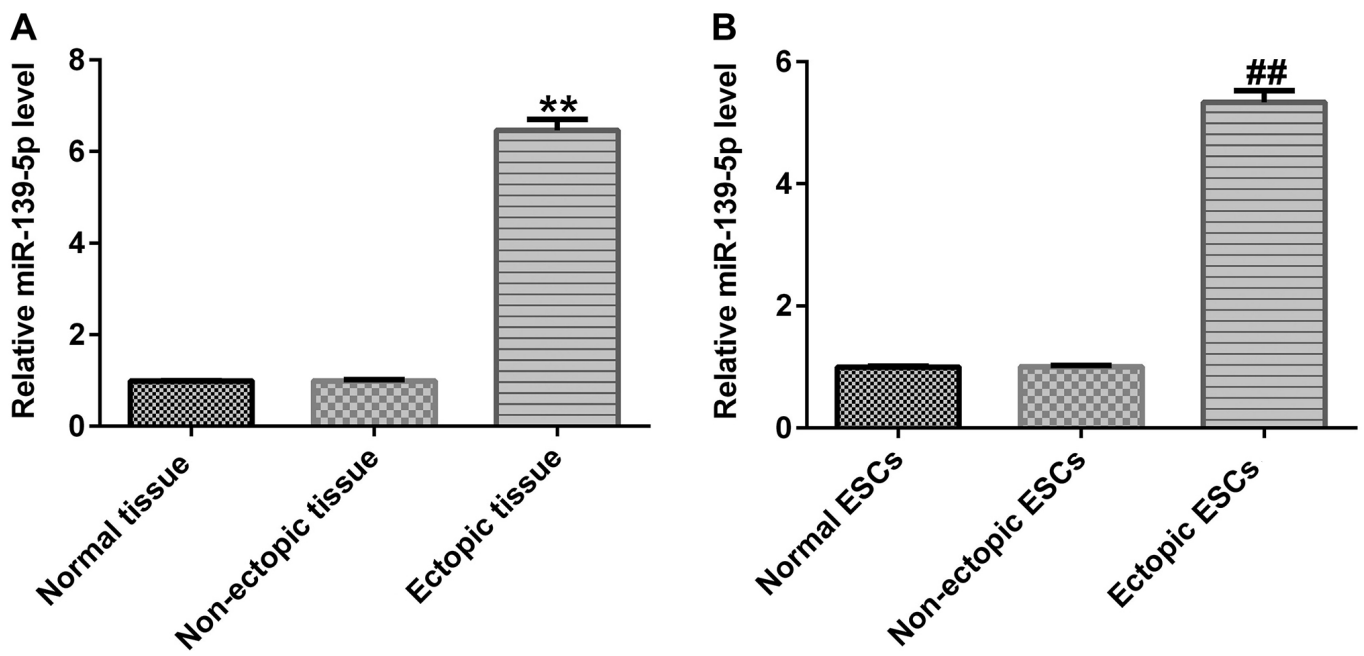

Figure 1. miR-139-5p expression levels in endometrial tissues and ESCs. Reverse transcription-quantitative PCR was performed to analyze miR-139-5p expression levels in (A) endometrial tissues and (B) ESCs. ${ }^{* *} \mathrm{P}<0.01$ vs. normal tissue; ${ }^{\# \#} \mathrm{P}<0.01$ vs. normal ESCs. miR, microRNA; ESC, endometrial stromal cells.

A

\begin{tabular}{l|cc} 
Position 879-885 of BBC3 3' UTR & 5, & $\ldots$ ACUGUAUAUGCGCUGCUGUAGAU. .. \\
hsa-miR-139-5p & 3, & UGACCUCUGUGCACGUGACAUCU
\end{tabular}

B

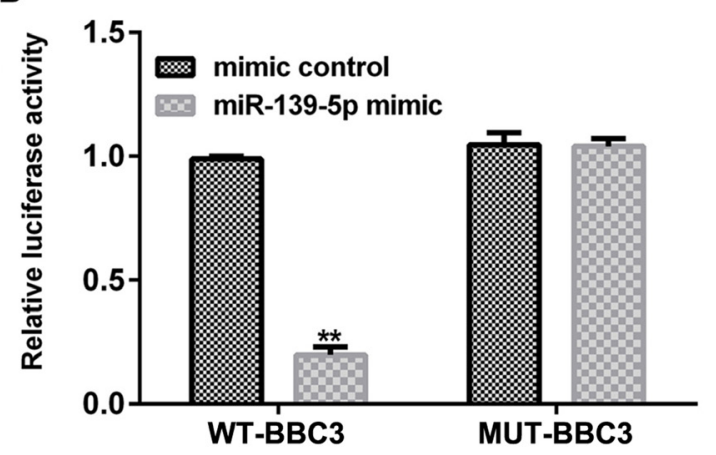

Figure 2. miR-139-5p directly targets BBC3. (A) Predicted WT miR-139-5p binding sites in the BBC3 3'-UTR. (B) 293T cells were co-transfected with WT/MUT-BBC3 3'-UTR reporter vector and miR-139-5p mimic/mimic control. Following $48 \mathrm{~h}$ of transfection, relative luciferase activity was analyzed using a dual luciferase reporter assay. ${ }^{* *} \mathrm{P}<0.01$ vs. mimic control (WT-BBC3). miR, microRNA; WT, wild-type; MUT, mutant; UTR, untranslated region; $\mathrm{BBC} 3, \mathrm{Bcl}-2$ binding component 3 .

observed in the relative luciferase activities of the MUT-BBC3 3'-UTRs in the cells co-transfected with the mimic control or miR-139-5p mimic (Fig. 2B). Taken together, these results suggested that BBC3 is a direct target of miR-139-5p.

BBC3 expression levels are downregulated in ectopic endometriosis tissues and ESCs. BBC3 mRNA expression levels in tissue samples and ESCs were determined via RT-qPCR analysis. The results demonstrated that BBC3 mRNA expression levels were significantly downregulated in ectopic endometrial tissues and ESCs compared with the normal endometrium tissues and ESCs, respectively (Fig. 3A and B). Furthermore, BBC3 protein expression levels were determined using western blotting. Similarly, BBC3 protein expression levels were markedly downregulated in ectopic tissues and
ESCs compared with the normal endometrium tissues and ESCs, respectively (Fig. 3C and D).

$B B C 3$ is negatively regulated by miR-139-5p. To determine the roles of miR-139-5p and BBC3 in endometriosis, ectopic ESCs were transfected with inhibitor control, miR-139-5p inhibitor, control-siRNA or BBC3-siRNA. mRNA and protein expression levels of miR-139-5p and BBC3 were detected via RT-qPCR and western blotting, respectively. The results demonstrated that transfection with the miR-139-5p inhibitor significantly downregulated miR-139-5p expression in ectopic ESCs compared with the inhibitor control group (Fig. 4A). Similarly, compared with the control-siRNA group, BBC3-siRNA significantly downregulated BBC3 mRNA expression levels in ectopic ESCs (Fig. 4B). Conversely, transfection with the miR-139-5p inhibitor significantly upregulated BBC3 expression levels in ectopic ESCs compared with the inhibitor control group, which was reversed following co-transfection with BBC3-siRNA (Fig. 4C and D).

$B B C 3$ reverses the effects of $m i R-139-5 p$ on viability, apoptosis, migration and invasion in ectopic ESCs. Flow cytometric analysis, and MTT and Transwell assays were performed to determine the potential underlying molecular mechanism of miR-139-5p in the regulation of cell apoptosis, viability, and migration and invasion in endometriosis. The results of the MTT assay demonstrated that transfection with the miR-139-5p inhibitor significantly decreased the viability of ectopic ESCs compared with the inhibitor control group, which was reversed following the combined knockdown of BBC3 (Fig. 5A). Similarly, the results of the Transwell assays indicated that the inhibitory effects of the miR-139-5p inhibitor on the migration and invasion of ectopic ESCs were suppressed following the knockdown of BBC3 (Figs. 5B and C, and S1 and S2). Furthermore, transfection with the miR-139-5p inhibitor significantly increased the levels of apoptosis in ectopic ESCs, which were partially reversed following co-transfection with BBC3-siRNA (Fig. 5D and E). 
A

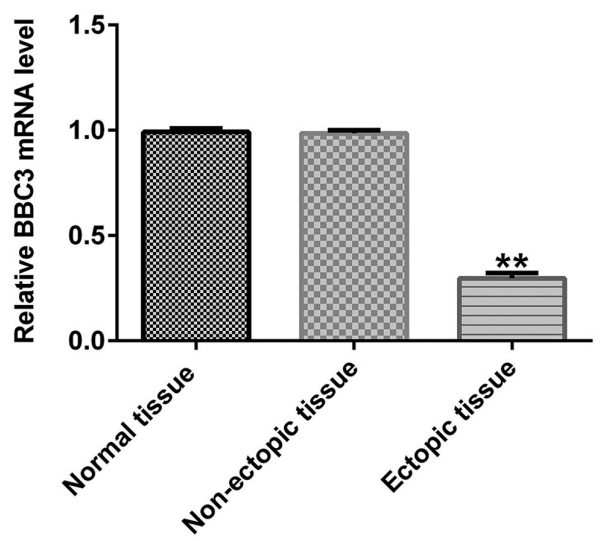

C

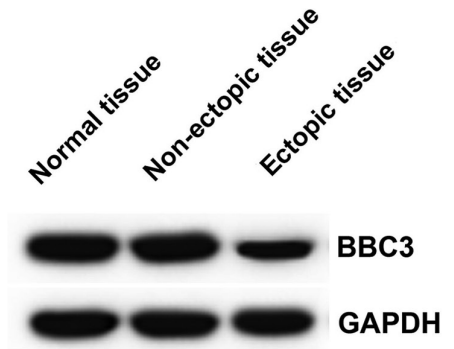

B

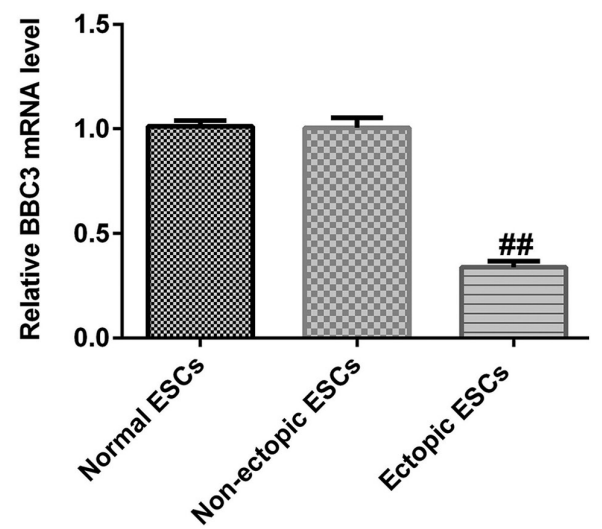

D

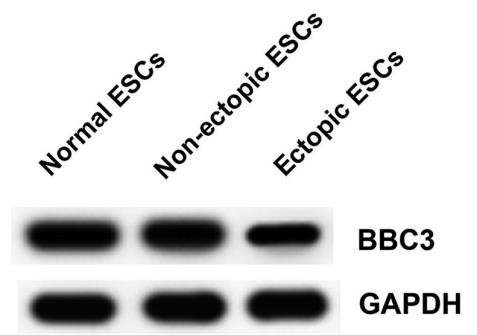

Figure 3. BBC3 expression levels in endometrial tissues and ESCs. Reverse transcription-quantitative PCR was performed to determine BBC3 mRNA expression levels in (A) endometrial tissues and (B) ESCs. Western blotting was performed to determine BBC3 protein expression levels in (C) endometrial tissues and (D) ESCs. ${ }^{* *} \mathrm{P}<0.01$ vs. normal tissue; ${ }^{\# \#} \mathrm{P}<0.01$ vs. normal ESCs. BBC3, Bcl-2 binding component 3; ESC, endometrial stromal cells.

A

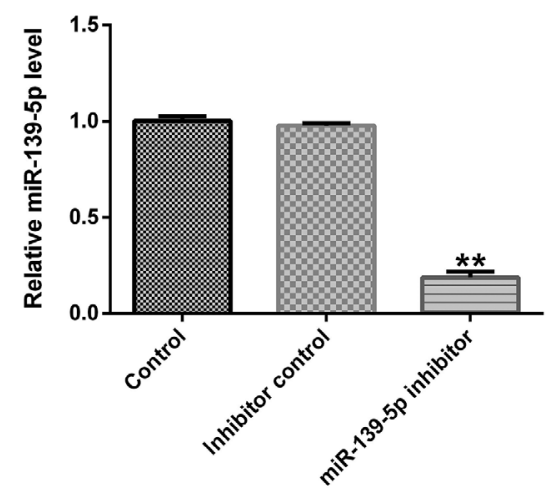

C

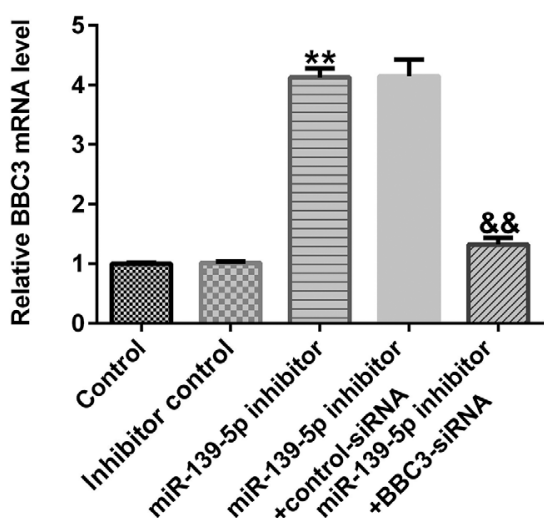

B

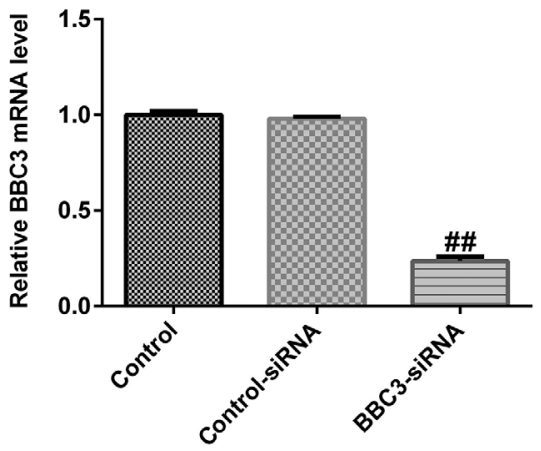

D

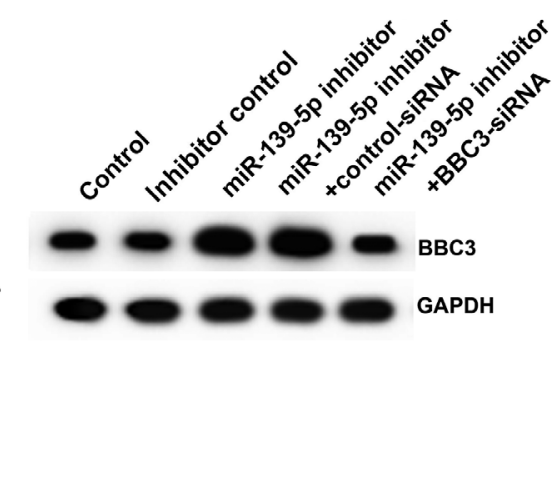

Figure 4. Transfection efficiency of miR-139-5p inhibitor and BBC3-siRNA in ectopic ESCs. Reverse transcription-quantitative PCR was performed to determine (A) miR-139-5p expression levels in ectopic ESCs transfected with the miR-139-5p inhibitor, (B) BBC3 mRNA expression levels in ectopic ESCs following transfection with BBC3-siRNA and (C) BBC3 expression levels in cells transfected with miR-139-5p inhibitor + BBC3-siRNA. (D) Western blotting was performed to determine BBC3 protein expression levels in ectopic ESCs following transfection with miR-139-5p inhibitor + BBC3-siRNA. " $\mathrm{P}<0.01$ vs. inhibitor control; ${ }^{\# \#} \mathrm{P}<0.01$ vs. control-siRNA; ${ }^{\&}{ }^{\&} \mathrm{P}<0.01$ vs. miR-139-5p inhibitor + control-siRNA. miR, microRNA; BBC3, Bcl-2 binding component 3; siRNA, small interfering RNA; ESC, endometrial stromal cells. 
A

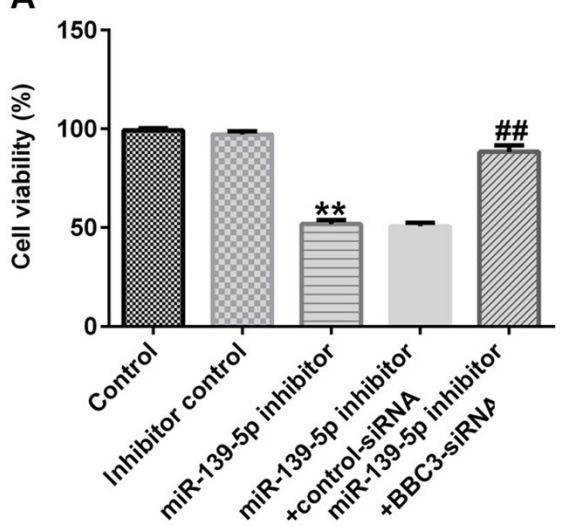

B

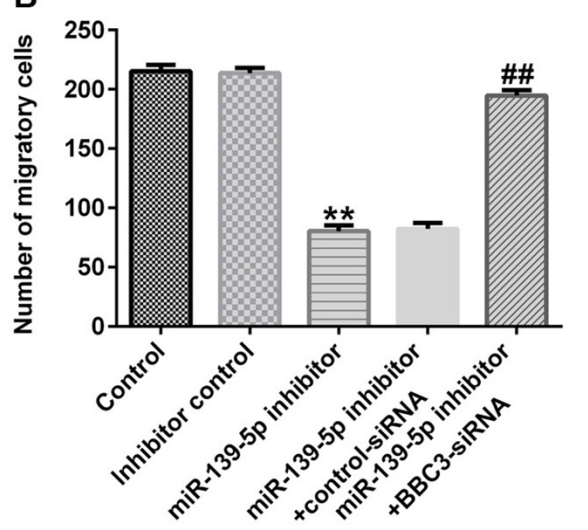

C

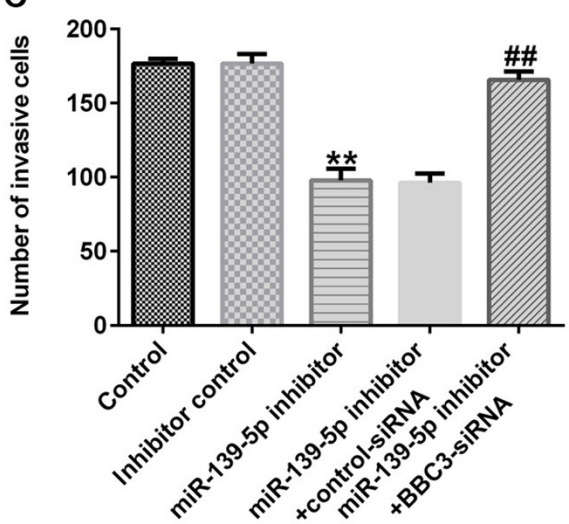

E

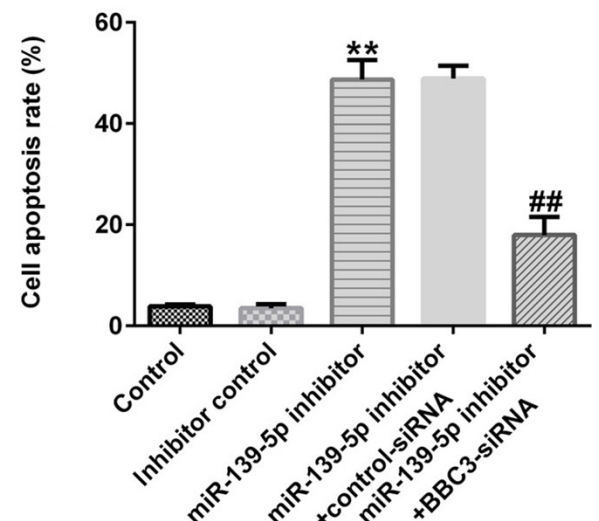

D

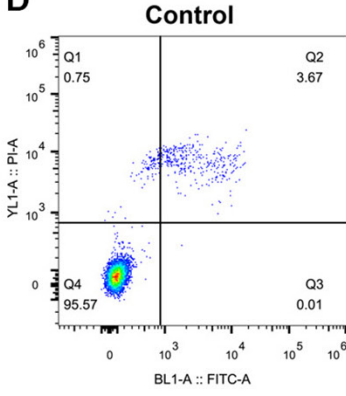

miR-139-5p inhibitor +control-siRNA

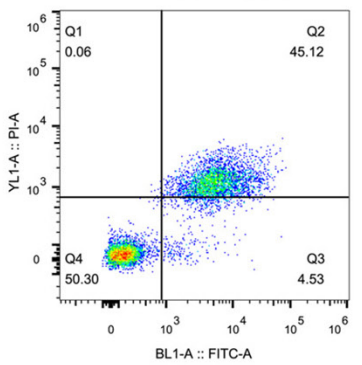

Inhibitor control

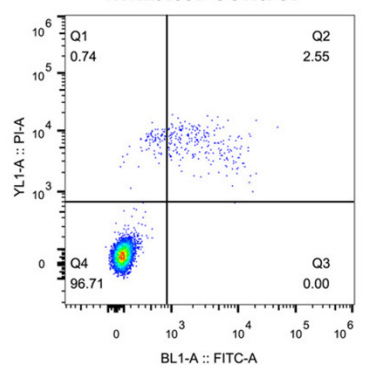

miR-139-5p inhibito +BBC3-siRNA

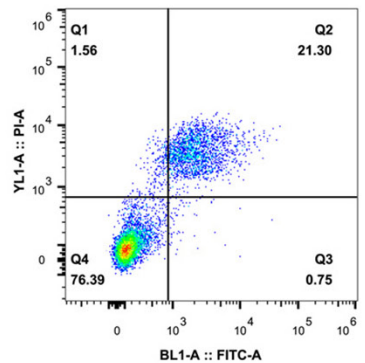

miR-139-5p inhibitor

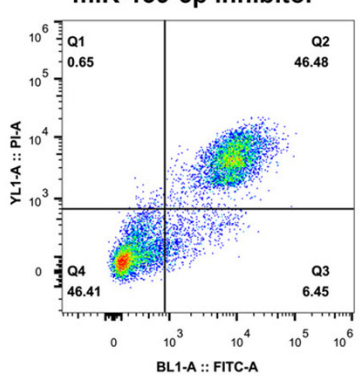

02
46.48

Figure 5. Effects of the miR-139-5p inhibitor and BBC3-siRNA on the viability, migration, invasion and apoptosis of ectopic ESCs. (A) MTT assay was performed to assess the viability of transfected ectopic ESCs. Transwell assays were performed to assess the (B) migration and (C) invasion of transfected ectopic ESCs. (D) Flow cytometry analysis was performed to determine the apoptotic rates of transfected ectopic ESCs. (E) Quantification of the apoptotic rates from part (D). ${ }^{* *} \mathrm{P}<0.01$ vs. inhibitor control; ${ }^{\# \prime} \mathrm{P}<0.01$ vs. miR-139-5p inhibitor + control-siRNA. miR, microRNA; BBC3, Bcl-2 binding component 3; siRNA, small interfering RNA; ESC, endometrial stromal cells.

\section{Discussion}

A common histological feature of endometriosis is the presence of dense fibrous tissue around the endometrial glands and stroma (22). Furthermore, the migration and invasion of ectopic ESCs were found to be similar to that observed of malignant cancer cells (5). Previous studies have demonstrated that the aberrant expression of miRNAs was implicated in the progression of endometriosis $(23,24)$. Both the migratory and invasive abilities of endometriotic cells were discovered to play key roles in the development of endometriosis (25). In fact, miR-139-5p was found to serve an important role in the progression and development of several types of tumor. For example, miR-139-5p expression levels were reported to be downregulated in endometrial, colorectal and small cell lung cancer $(17,26,27)$. Conversely, Rekker et al (19) reported that miR-139-5p expression was significantly upregulated in ectopic endometrial tissues and ESCs. The present study extracted ectopic, non-ectopic and normal ESCs from related endometrial samples in premenopausal women. Consistent with the latter finding, the results of the present study demonstrated that miR-139-5p expression levels were significantly upregulated in ectopic endometrial samples and ESCs, suggesting that miR-139-5p may be involved in the progression of endometriosis.

It is well-known that miRNAs exert their biological function by binding to the 3'-UTR of target genes, and they have been found to be involved in the development of endometriosis $(6,7,23,24)$. To further investigate the underlying molecular mechanism of miR-139-5p in the development of endometriosis, BBC3 was verified as a direct target of miR-139-5p and was negatively regulated by miR-139-5p in endometriosis. BBC3, also known as p53 upregulated modulator of apoptosis, is a member of the Bcl-2 family, which is involved in regulating cell apoptosis (28). BBC3 is a positive regulator of cell death and induces apoptosis when cells are appropriately stimulated $(29,30)$. The results of the present study demonstrated that the expression levels of $\mathrm{BBC} 3$ were downregulated in ectopic 
endometrial tissues and ESCs. The migratory and invasive properties of ectopic ESCs were found to be similar to those of malignant cancer cells (5). Thus, to determine whether miR-139-5p affected the apoptosis, viability, migration and invasion of ectopic ESCs by directly targeting BBC3, a series of experiments were performed in ectopic ESCs following the knockdown of miR-139-5p or BBC3 expression. The results demonstrated that transfection with the miR-139-5p inhibitor suppressed the viability, migration and invasion of ectopic ESCs, and induced cell apoptosis; however, all these effects were reversed following the knockdown of BBC3. The data indicated that the miR-139-5p inhibitor may negatively regulate viability, migration and invasion, while inducing apoptosis in ectopic ESCs by targeting BBC3 to participate in the occurrence and development of endometriosis.

However, there were several limitations to the present study. First, the sample size used in the study was small and larger sample sizes should be used to further investigate the expression levels of miR-139-5p in patients with endometriosis and ectopic ESCs. In addition, normal tissue samples collected from patients with hysteromyoma were used as the control samples in this study. Unfortunately, fibroids can alter the local environment in the uterine cavity and the patients are typically older (31), therefore, selecting the endometrial tissue of patients with uterine fibroids as the control group may not be ideal. Moreover, the current study focused on the effect of miR-139-5p on ectopic ESCs. To further verify the present results, the expression levels of miR-139-5p in endometrial epithelial cells and endometrial stromal cells in normal, non-ectopic and ectopic samples should be further analyzed, which is an aim of future studies.

In conclusion, the results of the present study indicated that miR-139-5p regulated the viability, migration, invasion and apoptosis of ectopic ESCs by directly targeting BBC3. These findings suggested that miR-139-5p may represent a potential target for the treatment of endometriosis and provide novel opportunities for clinical therapies for endometriosis.

\section{Acknowledgements}

Not applicable.

\section{Funding}

No funding was received.

\section{Availability of data and materials}

The datasets used and/or analyzed during the current study are available from the corresponding author on reasonable request.

\section{Authors' contributions}

LF contributed to the study design, data collection, statistical analysis, data interpretation and manuscript preparation. XC, $\mathrm{SZ}$ and YC contributed to the data collection and statistical analysis. YY contributed to the data collection, statistical analysis and manuscript preparation. LF and YY confirmed the authenticity of all the raw data. All authors read and approved the final manuscript.

\section{Ethics approval and consent to participate}

The present study was approved by the Ethics Committee of The Second Affiliated Hospital of Shenzhen University (Shenzhen, China) and written informed consent was provided by all participants prior to the study start.

\section{Patient consent for publication}

Not applicable.

\section{Competing interests}

The authors declare that they have no competing interests.

\section{References}

1. Muñoz-Hernando L, Muñoz-Gonzalez JL, Marqueta-Marques L, Alvarez-Conejo C, Tejerizo-García Á, Lopez-Gonzalez G, Villegas-Muñoz E, Martin-Jimenez A and Jiménez-López JS: Endometriosis: Alternative methods of medical treatment. Int J Women's Health 7: 595-603, 2015.

2. Marí-Alexandre J, Barceló-Molina M, Olcina-Guillem M, García-Oms J, Braza-Boïls A and Gilabert-Estellés J: MicroRNAs: New players in endometriosis. World J Obstet Gynecol 5: 28-38, 2016.

3. Yerlikaya G, Balendran S, Pröstling K, Reischer T, Birner P, Wenzl R, Kuessel L, Streubel B and Husslein H: Comprehensive study of angiogenic factors in women with endometriosis compared to women without endometriosis. Eur J Obstet Gynecol Reprod Biol 204: 88-98, 2016.

4. Ferrero S, Barra F and Leone Roberti Maggiore U: Current and emerging therapeutics for the management of endometriosis. Drugs 78: 995-1012, 2018.

5. Diao R, Wei W,Zhao J, Tian F, Cai X and Duan YG: CCL19/CCR7 contributes to the pathogenesis of endometriosis via PI3K/Akt pathway by regulating the proliferation and invasion of ESCs. Am J Reprod Immunol 78: e12744, 2017.

6. Hébert SS, Wang WX, Zhu Q and Nelson PT: A study of small RNAs from cerebral neocortex of pathology-verified Alzheimer's disease, dementia with lewy bodies, hippocampal sclerosis, frontotemporal lobar dementia, and non-demented human controls. J Alzheimers Dis 35: 335-348, 2013.

7. Li M, Marin-Muller C, Bharadwaj U, Chow KH, Yao Q and Chen C: MicroRNAs: Control and loss of control in human physiology and disease. World J Surg 33: 667-684, 2009.

8. Farazi TA, Spitzer JI, Morozov P and Tuschl T: miRNAs in human cancer. J Pathol 223: 102-115, 2010.

9. Sayed AS, Xia K, Salma U, Yang T and Peng J: Diagnosis, prognosis and therapeutic role of circulating miRNAs in cardiovascular diseases. Heart Lung Circ 23: 503-510, 2014.

10. Santamaria $X$ and Taylor H: MicroRNA and gynecological reproductive diseases. Fertil Steril 101: 1545-1551, 2014.

11. Xia G, Bao L, Gao W, Liu S, Ji K and Li J: Differentially expressed miRNA in inflammatory mucosa of chronic rhinosinusitis. J Nanosci Nanotechno 15: 2132-2139, 2015.

12. Prislei S, Martinelli E, Mariani M, Raspaglio G, Sieber S, Ferrandina G, Shahabi S, Scambia G and Ferlini C: miR-200c and $\mathrm{HuR}$ in ovarian cancer. BMC Cancer 13: 72, 2013.

13. He W, Huang H, Xie Q, Wang Z, Fan Y, Kong B, Huang D and Xiao Y: miR-155 knockout in fibroblasts improves cardiac remodeling by targeting tumor protein p53-inducible nuclear protein 1. J Cardiovasc Pharmacol Ther 21: 423-435, 2016.

14. Lee JW, Park YA, Choi JJ, Lee YY, Kim CJ, Choi C, Kim TJ, Lee NW, Kim BG and Bae DS: The expression of the miRNA-200 family in endometrial endometrioid carcinoma. Gynecol Oncol 120: 56-62, 2011.

15. Hsu CY,Hsieh TH, Tsai CF, Tsai HP, Chen HS, Chang Y, Chuang HY, Lee JN, Hsu YL and Tsai EM: miRNA-199a-5p regulates VEGFA in endometrial mesenchymal stem cells and contributes to the pathogenesis of endometriosis. J Pathol 232: 330-343, 2014.

16. Song M, Yin Y, Zhang J, Zhang B, Bian Z, Quan C, Zhou L, $\mathrm{Hu}$ Y, Wang Q, Ni S, et al: miR-139-5p inhibits migration and invasion of colorectal cancer by downregulating AMFR and NOTCH1. Protein Cell 5: 851-861, 2014. 
17. Liu H, Yin Y, Hu Y, Feng Y, Bian Z, Yao S, Li M, You Q and Huang Z: miR-139-5p sensitizes colorectal cancer cells to 5-fluorouracil by targeting NOTCH-1. Pathol Res Pract 212: 643-649, 2016.

18. van den Akker EK, Dor FJ, IJzermans JN and de Bruin RW: MicroRNAs in kidney transplantation: Living up to their expectations. J Transplant 2015: 354826, 2015.

19. Rekker K, Tasa T, Saare M, Samuel K, Kadastik Ü, Karro H, Götte M, Salumets A and Peters M: Differentially-expressed miRNAs in ectopic stromal cells contribute to endometriosis development: The plausible role of miR-139-5p and miR-375. Int J Mol Sci 19: 3789, 2018.

20. Liu H, Zhang Z, Xiong W, Zhang L, Xiong Y, Li N, He H, Du Y and Liu Y: Hypoxia-inducible factor- $1 \alpha$ promotes endometrial stromal cells migration and invasion by upregulating autophagy in endometriosis. Reproduction 153: 809-820, 2017.

21. Livak KJ and Schmittgen TD: Analysis of relative gene expression data using real-time quantitative PCR and the 2(-Delta Delta C(T)) method. Methods 25: 402-408, 2001.

22. Apostol R, Sirota I, Mrkaic A and Nezhat FR: Benign ovarian tumors and endometriosis (106). Obstet Gynecol 125: S39-S40, 2015.

23. Olson MR, Vadlapatla NM, Khoo SK, Gadisetti C, Resau JH and Fazleabas AT: Early changes in micro RNA (miRNA) expression in the eutopic endometrium (EUE) in a baboon model of induced endometriosis. Fertil Steril 94 (Suppl): S200, 2010.
24. Bjorkman S and Taylor HS: MicroRNAs in endometriosis: Biological function and emerging biomarker candidates. Bio Reprod 100: 1135-1146, 2019.

25. Qiu JJ, Lin YY, Tang XY, Ding Y, Yi XF and Hua KQ: Extracellular vesicle-mediated transfer of the IncRNA-TC0101441 promotes endometriosis migration/invasion. Exp Cell Res 388: 111815, 2020.

26. Liu J, Li C, Jiang Y, Wan Y, Zhou S and Cheng W: Tumor-suppressor role of miR-139-5p in endometrial cancer. Cancer Cell Int 18: 51, 2018.

27. Liu H, Zhang G, Guo L and Tan S: Expression of miR-139-5p in small cell lung cancer tissue and its clinical significance. J Jilin Univ 42: 942-948, 2016.

28. Harder JM and Libby RT: BBC3 (PUMA) regulates developmental apoptosis but not axonal injury induced death in the retina. Mol Neurodegener 6: 50, 2011.

29. Nakano K and Vousden KH: PUMA, a novel proapoptotic gene, is induced by p53. Mol Cell 7: 683-694, 2001

30. Yu J and Zhang L: PUMA, a potent killer with or without p53. Oncogene 27 (Suppl 1): S71-S83, 2008.

31. De La Cruz MS and Buchanan EM: Uterine fibroids: Diagnosis and treatment. Am Fam Physician 95: 100-107, 2017.

(i) (3) This work is licensed under a Creative Commons Attribution-NonCommercial-NoDerivatives 4.0 International (CC BY-NC-ND 4.0) License. 